nephron

Practice
Nephron 2019;143:170-173

DOI: $10.1159 / 000501748$
Received: April 3, 2019

Accepted after revision: June 25, 2019

Published online: August 8, 2019

\title{
Acute Kidney Injury and Fluid Resuscitation in Septic Patients: Are We Protecting the Kidney?
}

\author{
Jonathan Montomoli ${ }^{a, b} \quad$ Abele Donati $^{a} \quad$ Can Ince $^{b}$ \\ ${ }^{a}$ Anesthesia and Intensive Care, Department of Biomedical Sciences and Public Health, Università Politecnica delle Marche,

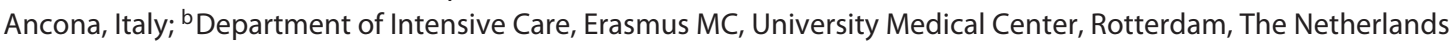

\section{Keywords}

Acute kidney injury · Critical care · Fluid therapy · Sepsis

\begin{abstract}
Acute kidney injury (AKI) is a common complication in critically ill patients, especially among septic patients. Sepsis and hypovolemia are the 2 most frequent etiologies of AKI in intensive care units and frequently coexist in critically ill patients. Effective fluid resuscitation is crucial for the stabilization of sepsis-induced tissue hypoperfusion or septic shock. However, the lack of a goal-directed therapy targeting kidney oxygenation prevents from optimization of the fluid therapy with regard to improvement of renal oxygen delivery and extraction. Similarly, fluid administration as all therapeutic actions carries adverse effects such as the activation of cytokines, disruption of the capillary glycocalyx, and adverse effects on kidney metabolism and oxygenation. Moreover, a positive fluid balance is associated with an increased risk of $\mathrm{AKI}$ and is a negative predictor for recovery of renal function. The role of fluid resuscitation on kidney injury stems from the high renal vulnerability to hypoxemic injury. Indeed, fluids have a poor oxygen solubility and hemodilution decreases blood viscosity both promoting intrarenal shunting and heterogeneity with a decreased capillary density and enhanced intrarenal cortex and medullary hypoxia. The development of physiological biomarkers that are able
\end{abstract}

\begin{tabular}{ll}
\hline KARGER & $\begin{array}{l}\text { (c) } 2019 \text { The Author(s) } \\
\text { Published by S. Karger AG, Basel }\end{array}$ \\
E-Mail karger@karger.com & This article is licensed under the Creative Commons Attribution- \\
www.karger.com/nef & $\begin{array}{l}\text { NonCommercial-NoDerivatives 4.0 International License (CC BY- } \\
\text { NC-ND) (http://www.karger.com/Services/OpenAccessicense). } \\
\text { Usage and distribution for commercial purposes as well as any dis- } \\
\text { tribution of modified material requires written permission. }\end{array}$
\end{tabular}

to detect the early development of AKI specifically aimed at the identification of renal microcirculatory dysfunctions should form a valuable contribution to monitoring therapeutic modalities.

(c) 2019 The Author(s)

Published by S. Karger AG, Basel

\section{Introduction}

Nearly 2 centuries ago, oliguria was recognized as a sign of renal failure and serum creatinine as marker of renal function. Despite that, their standardization of acute kidney injury (AKI) using serum creatinine and urinary output did not occur until the description of the Risk, Injury, Failure, Loss, End-stage criteria in 2004, where a urinary output of $<0.5 \mathrm{~mL} / \mathrm{kg} / \mathrm{h}$ for $>6 \mathrm{~h}$ was introduced as an alternative criterion to a rise in serum creatinine by 1.5 -fold from baseline. This definition has evolved to the AKI Network classification in 2007 and to the Kidney Disease Im-

Contribution from the AKI and CRRT 2019 Symposium at the 24th International Conference on Advances in Critical Care Nephrology, Manchester Grand Hyatt, San Diego, CA, USA, February 26 - March 1, 2019. This symposium was supported in part by the NIDDK funded University of Alabama at Birmingham-University of California San Diego O'Brien Center for Acute Kidney Injury Research (P30DK079337).
Prof. Can Ince, $\mathrm{PhD}$

Department of Intensive Care, Erasmus MC

University Medical Center, 's-Gravendijkwal 230

NL-3015 CE Rotterdam (The Netherlands)

E-Mail c.ince@erasmusmc.nl 
proving Global Outcomes classification in 2012. The incidence of AKI varies between one-third to two-thirds of patients admitted to intensive care units (ICUs) when defined by the sensitive Risk, Injury, Failure, Loss, End-stage, AKI Network, or Kidney Disease Improving Global Outcomes criteria and it is increasing over time as is the use of renal replacement therapy (RRT) [1]. Variation in AKI incidence and severity reported in critically ill patients may stem from baseline patients' characteristics, attitudes towards initiation of RRT, more consistent recording in administrative databases and the length of the observation period. The pathophysiology of AKI in septic patients is likely to be multifactorial involving hemodynamic and microcirculatory mechanisms that lead to impaired tissue oxygenation. However, the lack of accurate and sensitive diagnostic criteria for metabolic renal impairment hampers the prevention of AKI in ICU. Furthermore, the optimization of kidney perfusion is prevented by the lack of a goal-directed therapy for kidney oxygenation.

The aim of this brief review is to examine the rationale for actual fluid management in septic patients and identify some iatrogenic mechanisms that may contribute to AKI development.

\section{AKI in ICUs}

Among critically ill patients AKI may already be present at ICU admission (community-acquired) or develop during hospital stay (hospital-acquired). This second type has been shown to be associated with worse prognosis and its pathogenesis is most often iatrogenic. The 2 most frequent etiologies of AKI in ICU are sepsis and hypovolemia and frequently the 2 are associated. Volume depletion is a very frequent situation in ICU patients and is a well-known risk factor for AKI. On the one hand, the correction of volume deficit will prevent the extension of kidney injury and facilitate renal function recovery. On the other hand, fluid administration may lead to adverse events including tissue edema and anemia. Moreover, the primary pathophysiologic phenomenon in septic shock is vasoplegia, causing a state of hypotension not corrected by fluid administration but rather by vasoconstricting agents. Establishing the right fluid volume to be administered in septic patients is also a challenge. Finally, hemodynamic monitoring helps physicians to identify patients likely to be fluid responsiveness but seems to be insufficient to minimize the development of AKI and not provide information about the response of the renal microcirculation to fluid administration.

AKI and Sepsis

\section{Kidney Metabolism}

Considering its multiple etiologies, variable pathogenesis and diverse outcomes, AKI may be described as a syndrome characterized by a rapid deterioration of kidney function occurring within hours or days that may lead to the need of temporary or chronic RRT. The highly complex architecture of the renal microvasculature, the need to meet a high energy demand, and the fact that the kidney is borderline ischemic make the kidney a highly vulnerable organ to hypoxemic injury. In particular, approximately $90 \%$ of kidney oxygen requirement seems to be used for ATP production needed for $\mathrm{Na} / \mathrm{K}$ pump function. The mechanism accounting for the larger part of the oxygen consumption is the tubular sodium reabsorption, that is already exposed to ischemic damage in critically ill patients. Under normal, steady-state conditions, oxygen supply to the renal tissues is well regulated; however, under septic conditions, the delicate balance between oxygen supply and demand is disturbed due to renal microvasculature dysfunction [2]. The reduction in glomerular filtration rate secondary to hypotension related to hypovolemia and decreased cardiac output are the mechanisms considered to be responsible for oliguria and raised serum creatinine. However, in the early phase of sepsis, where fluids are being administered during the development or established AKI, an increased cardiac output underlies the development of a "hyperdynamic state".

\section{Fluid Resuscitation and the Kidney}

The need for prompt fluid resuscitation in the process of restoring euvolemia and systemic hemodynamics in septic patients is well established. However, a common response to an episode of hypotension in ICU is, nevertheless, the administration of fluids despite growing evidence showing that the cardiac output does not always increase in most critically ill patients when challenged with a fluid bolus according to fluid responsiveness criteria. In addition, the exact amount and precise timing of tapering such resuscitation is not established. Specifically, with regard to AKI, fluid resuscitation is considered a preventive measure based on the theory that restoration of circulating volume will improve renal perfusion. Pranskunas et al. [3] have examined changes in sublingual microcirculation before and after fluid administration in patients with clinical signs of impaired organ perfusion. They found that fluid administration was able to improve microcirculatory flow in patients with impairment at the baseline but with 
no impact on capillary density both in patients with and without microcirculatory flow impairment at the baseline. Accordingly, signs of impaired organ perfusion improved only among those patients with microcirculatory dysfunction at baseline but not in those with normal perfusion. These changes were independent of effects of fluid resuscitation on stroke volume which increased in all subjects. In other studies, the improvement of hemodynamic macrovascular parameters resulted in restoration, decreased or even unaffected cortical renal perfusion during septic shock [4]. Furthermore, it should be considered that fluids are not different from drugs and carry side effects such as the activation of cytokines, shedding of the capillary glycocalyx, and adversely affecting the capacity of the kidney to adequately filter excess fluid and nitrogenous waste. The recently released "hour-1 bundle" confirms that fluid resuscitation should be performed in septic patients with hypotension or lactate $\geq 4 \mathrm{mmol} / \mathrm{L}$ with $30 \mathrm{~mL} / \mathrm{kg}$ of crystalloids previously reported in the "3-h bundle" [5]. Despite being reported as strong recommendation, the quality of reported evidence is low and not supported by clinical studies. On the contrary, in adult patients admitted to the ICU with sepsis, a positive fluid balance after the first day was associated with an increased risk of AKI [6]. Not only does fluid overload increase mortality, but excess fluid has also been showed to be a negative predictor for recovery of renal function [1]. However, it is impossible to account for all potential confounders and it is difficult to prove whether fluid overload directly causes adverse outcomes or, vice versa, where critical illness itself is responsible for fluid overload. Fluid administration causes increased workload for the kidneys because of increased filtration of sodium chloride leading to increased reabsorption activity, and increased oxygen consumption of the tubular cells. Finally, although current international guidelines from the Surviving Sepsis Campaign recommend crystalloids for initial resuscitation and subsequent volume replacement with albumin "when patients require substantial amount of crystalloids", the debate on which type of fluid to use in the resuscitation phase is still open. A recent meta-analysis including 55 randomized clinical trials reported that crystalloids were less effective than colloids in stabilizing resuscitation endpoints such as mean arterial pressure, cardiac index, and central venous pressure [7]. Moreover, although the primary outcome in the CRISTAL trial showed a not significant lower 30-day mortality in the colloids group in comparison to the crystalloid group ( $p$ value 0.26 ), 90-day mortality was significantly lower ( $p$ value 0.03 ) when colloids were used for fluid resuscitation [8]. Inconsistences with studies reporting dif- ferent findings may stem from different patients' volemic state at the time of randomization [9]. Despite the increased use of balanced solutions, $\mathrm{NaCl} 0.9 \%$ is probably still the most used crystalloid solution for fluid management [10]. This is despite the well-documented adverse effects of the use of $\mathrm{NaCl} 0.9 \%$ [11]. Finally, existing evidence supporting albumin use in patients with septic shock comes from the ALBIOS study where albumin administration was used to replenish albumin instead of using it for treating hypovolemia in a similar manner to the manner in which synthetic colloids are used [12].

\section{AKI from a Microcirculatory Point of View}

Microcirculatory dysfunction in sepsis is characterized by heterogeneous abnormalities in renal blood flow in which some capillaries are under-perfused, while others have normal or abnormally high blood flow. Although the issue is still a controversy, histopathological studies have shown that sepsis or septic shock can lead to areas of ischemia of tubular cells mainly located at the cortex microcirculatory level because of hypoxia and the overproduction of reactive oxygen and nitrogen species and cytokines [13]. These heterogeneous conditions associated with sepsis can also be the result of therapy such as the administration of fluids. Thus, although fluid resuscitation can normalize the renal arterial flow, it can cause heterogeneous microcirculatory flow in the renal cortex, resulting in a pattern of hypoxic areas next to normoxic areas. As a consequence, hypoxia contributes to renal oxygen extraction dysfunction and production of reactive oxygen species that can further contribute to kidney injury [14]. Indeed, findings from Legrand et al. [15] suggested that half of the oliguric patients in the ICUs are not renal responders to fluid challenge although they show a macrohemodynamic response. Additionally, sepsis-induced endothelial dysfunctions such as microvascular thrombosis, endothelial injury, and shedding of the endothelial glycocalyx further promote abnormal microcirculation, areas of hypoxemia, and increased capillary leak decreasing the potential benefits of fluids. In addition, fluid administration itself may increase inflammation contributing to endothelial degradation promoting rolling and adhesion of leucocytes and increased platelet adhesion. Yet, hemodilution caused by fluids leading to anemia is probably the most deleterious effect associated with fluid administration. Indeed, besides the poor oxygen solubility of fluids, hemodilution decreases blood viscosity promoting intrarenal shunting and heterogeneity with a de- 
creased capillary density and enhances microcirculatory cortex and medullary hypoxia. Hemodilution reduces renal tissue oxygenation causing reduction in oxygen extraction and ineffective sodium reabsorption associated with a loss of tubular polarity. In support of this notion, animal data suggest that blood transfusion may improve renal microvascular oxygenation and function superior to resuscitation with fluids [16] and improved microcirculatory perfusion in cardiosurgical patients [17].

\section{Conclusion}

AKI is a frequent complication in septic patients and optimization of fluid management is fundamental in order to limit the detrimental role of fluids on renal metabolism. In particular, during the first phases, fluid resuscitation should take into account the etiology of AKI, the volume status of the patients, the type of fluid, and its infusion rate. Finally, the lack of available tools at the bedside of patients able to provide information regarding renal microcirculation, prevent from identifying therapeutic target for fluid management specific for renal oxygen delivery optimization. Recent animal studies have shown that sublingual microcirculatory alterations closely follow renal microcirculatory alterations in septic shock and during resuscitation, whereas systemic hemodynamic alterations that normalize during fluid resuscitation do not [18]. These results suggest that the identification of alterations in sublingual microcirculation may be used as a surrogate for renal microcirculatory alterations indicating a kidney at risk for developing AKI [19].

\section{Disclosure Statement}

C.I. has received honoraria and independent research grants from Fresenius-Kabi, Bad Homburg, Germany; Baxter Health Care, Deerfield, Illinois; AM-Pharma, Bunnik, The Netherlands; Novartis, Basel, Switzerland; Hutchinson, Hutchinson, Minnesota; B. Braun, Melsungen, Germany; Covidien, Dublin, Ireland; and Eli Lilly, Indianapolis, Indiana. He is the inventor of SDF technology, which is commercialized by MicroVision Medical. He has been a consultant for this company in the past, but he has broken all contact with this company for more than 4 years, and he has no competing interests other than his commitment to promote the importance of the microcirculation in the care of critically ill patients.

\section{References}

1 Bellomo R, Ronco C, Mehta RL, Asfar P, Boisramé-Helms J, Darmon M, et al. Acute kidney injury in the ICU: from injury to recovery: reports from the 5th Paris International Conference. Ann Intensive Care. 2017 Dec;7(1):49.

2 Ergin B, Kapucu A, Demirci-Tansel C, Ince C. The renal microcirculation in sepsis. Nephrol Dial Transplant. 2015 Feb;30(2):169-177.

3 Pranskunas A, Koopmans M, Koetsier PM, Pilvinis V, Boerma EC. Microcirculatory blood flow as a tool to select ICU patients eligible for fluid therapy. Intensive Care Med. 2013 Apr;39(4):612-9.

4 Harrois A, Grillot N, Figueiredo S, Duranteau $\mathrm{J}$. Acute kidney injury is associated with a decrease in cortical renal perfusion during septic shock. Crit Care. 2018 Jun;22(1):161.

5 Levy MM, Evans LE, Rhodes A. The Surviving Sepsis Campaign Bundle: 2018 Update. Crit Care Med. 2018 Jun;46(6):997-1000.

6 Salahuddin N, Sammani M, Hamdan A, Joseph M, Al-Nemary Y, Alquaiz R, et al. Fluid overload is an independent risk factor for acute kidney injury in critically Ill patients: results of a cohort study. BMC Nephrol. 2017 Feb;18(1):45.

7 Martin GS, Bassett P. Crystalloids vs. colloids for fluid resuscitation in the Intensive Care Unit: A systematic review and meta-analysis. J Crit Care. 2019 Apr;50:144-54.
8 Annane D, Siami S, Jaber S, Martin C, Elatrous $\mathrm{S}$, Declère AD, et al.; CRISTAL Investigators. Effects of fluid resuscitation with colloids vs crystalloids on mortality in critically ill patients presenting with hypovolemic shock: the CRISTAL randomized trial. JAMA. 2013 Nov;310(17):1809-17.

9 Montomoli J, Donati A, Ince C. Should Albumin be the Colloid of Choice for Fluid Resuscitation in Hypovolemic Patients? Berlin, Heidelberg: Springer Berlin Heidelberg; 2019, pp. 277-292.

10 Glassford NJ, French CJ, Bailey M, Mârtensson J, Eastwood GM, Bellomo R. Changes in intravenous fluid use patterns in Australia and New Zealand: evidence of research translating into practice. Crit Care Resusc. 2016 Jun;18(2):78-88.

11 Ince $C$, Groeneveld $A B$. The case for $0.9 \%$ $\mathrm{NaCl}$ : is the undefendable, defensible? Kidney Int. 2014 Dec;86(6):1087-95.

12 Caironi P, Tognoni G, Masson S, Fumagalli R, Pesenti A, Romero M, et al.; ALBIOS Study Investigators. Albumin replacement in patients with severe sepsis or septic shock. $\mathrm{N}$ Engl J Med. 2014 Apr;370(15):1412-21.

13 Ow CP, Ngo JP, Ullah MM, Hilliard LM, Evans RG. Renal hypoxia in kidney disease: cause or consequence? Acta Physiol (Oxf). 2018 Apr;222(4):e12999.
14 Clanton TL. Hypoxia-induced reactive oxygen species formation in skeletal muscle. J Appl Physiol (1985). 2007 Jun;102(6):2379-88.

15 Legrand M, Le Cam B, Perbet S, Roger C, Darmon $M$, Guerci $P$, et al.; support of the AZUREA network. Urine sodium concentration to predict fluid responsiveness in oliguric ICU patients: a prospective multicenter observational study. Crit Care. 2016 May;20(1):165

16 Zafrani L, Ergin B, Kapucu A, Ince C. Blood transfusion improves renal oxygenation and renal function in sepsis-induced acute kidney injury in rats. Crit Care. 2016 Dec;20(1):406.

17 Yuruk K, Almac E, Bezemer R, Goedhart P, de Mol B, Ince C. Blood transfusions recruit the microcirculation during cardiac surgery. Transfusion. 2011 May;51(5):961-7.

18 Lima A, van Rooij T, Ergin B, Sorelli M, Ince Y, Specht PA, et al. Dynamic Contrast-Enhanced Ultrasound Identifies Microcirculatory Alterations in Sepsis-Induced Acute Kidney Injury. Crit Care Med. 2018 Aug;46(8):1284-92.

19 Ince C, Boerma EC, Cecconi M, De Backer D, Shapiro NI, Duranteau J, et al.; Cardiovascular Dynamics Section of the ESICM. Second consensus on the assessment of sublingual microcirculation in critically ill patients: results from a task force of the European Society of Intensive Care Medicine. Intensive Care Med. 2018 Mar;44(3):281-99. 